\title{
Robust Stabilization for Markovian Jump Delta Operator Systems with Time-Varying Delays
}

\author{
Yinyin Chen and Minqing Xiao* \\ School of Mathematics and Computer Science, Fujian Normal University, Fuzhou 350007, P. R. China \\ *Corresponding author
}

\begin{abstract}
The robust stabilization problem is investigated for a class of delta operator formulated Markovian jump systems with time-varying delays. The aim is to design a state feedback controller which can make the closed-loop system stochastic asymptotically stable in delta domain. A sufficient condition of the existence of such controllers is obtained by the linear matrix inequality approach and a design procedure of such controllers is presented. Furthermore, the proposed method can unify some previous related continuous and discrete systems into the framework on operator systems. A numerical example is provided to demonstrate availability and efficiency of the design method.
\end{abstract}

Keywords-robust stochastic stabilization, Markovian jump system, delta operator system, time-varying delays

\section{INTRODUCTION}

Goodwin constructed delta operator instead of traditional shift operator for sampling continuous systems in [1]. The proposed method can unify some previous related results of the continuous and discrete systems into the frame-work of the delta operator systems framework. A class of system in delta domain has been investigated in [2-4]. The problem of system instability when the sampling time is fast can be solved by using delta operator model [5]. It also has been shown better numerical properties by using delta operator than using shift operator at high sampling rate [6].

The Markovian jump systems have finite modes, which may jump from one to another at different times and between different modes. Over the past years, much attention has been focused on problems of robust control for uncertain standard state-space systems, refer to [8] and so on. Furthermore, there are also some papers considering the discrete systems with time-varying delay [9].

However, to the best of our knowledge, there have been few papers on delta operator state feedback controller design problem for Markovian jump delta operator system with norm-bounded parameter uncertainties and time-varying delays. This problem is important and challenging in both theory and practice, which motivated us for this study.

\section{PROBLEM DESCRIPTION}

Given a probability space $(\Omega, F, P)$, where $\Omega$ is the sample space, $F$ is the algebra of event and $P$ is the probability measure defined on $F,\left\{\eta_{t_{k}}, t_{k} \geq 0\right\}$ is homogeneous, finite-state Markovian process with right continuous trajectories and taking values in a finite set
$S=\{1,2, \mathrm{~L} s\}$,with generator $\Lambda_{\delta}=\left(\lambda_{i j}\right)$. The transition probability from mode $i$ at time $t_{k}$ to the mode $j$ at time

$t_{k}+T_{. .} i, j \in S$, is $\operatorname{Pr}\left(\eta_{t_{k}+T}=j \mid \eta_{t_{k}}=i\right)=\left\{\begin{array}{l}\lambda_{i j} T, i \neq j \\ 1+\lambda_{i j} T, i=j\end{array}\right.$

Where the transition probability rates satisfy $\lambda_{i j} \geq 0$ for $i, j \in S$, $i \neq j$ and $\lambda_{i i}=-\sum_{j=1, j \neq i}^{s} \lambda_{i j}$. The Markov process transition rate matrix $\Lambda_{\delta}$ is defined by

$$
\Lambda_{\delta}=\frac{\Lambda_{z}(T)-\Lambda_{z}(0)}{T-0}=\left[\begin{array}{cccc}
\lambda_{11} & \lambda_{12} & \mathrm{~L} & \lambda_{1 s} \\
\lambda_{21} & \lambda_{22} & \mathrm{~L} & \lambda_{2 s} \\
\mathrm{M} & \mathrm{M} & \mathrm{O} & \mathrm{M} \\
\lambda_{s 1} & \lambda_{s 2} & \mathrm{~L} & \lambda_{\mathrm{ss}}
\end{array}\right]
$$

Where $\Lambda_{z}$ is the Markovian process transition probability matrix in z-domain and $\Lambda_{\delta}$ is the Markov process transition rate matrix in $\delta$-domain.

In this chapter, the following uncertain jump delta operator system with time-varying delays is considered:

$$
\begin{aligned}
\delta x\left(t_{k}\right)= & \left(A\left(\eta_{t_{k}}\right)+\Delta A\left(\eta_{t_{k}}, t_{k}\right)\right) x\left(t_{k}\right)+B\left(\eta_{t_{k}}\right) u\left(t_{k}\right) \\
& +\left(A_{d}\left(\eta_{t_{k}}\right)+\Delta A_{d}\left(\eta_{t_{k}}, t_{k}\right)\right) x\left(t_{k}-d(k)\right)
\end{aligned}
$$

Where $x\left(t_{k}\right) \in R^{n}$ is the state variable; $u\left(t_{k}\right) \in R^{m}$ is control input. The time-delay $d(k)=\hat{n} T$ is a time-varying function that satisfies $0 \leq d_{m} \leq d(k) \leq d_{M}$ with $d_{m}=n_{m} T$ and $d_{M}=n_{M} T$, $n_{m}$ and $n_{M}$ are two known positive integers, from which we let $n_{m} \leq \hat{n} \leq n_{M}$. For notational simplicity, in the sequel, for $\eta_{t_{k}}=i \in S$, we let

$$
\begin{gathered}
A\left(\eta_{t_{k}}\right)=A_{i} A_{i}\left(t_{k}\right)=A\left(\eta_{t_{k}}\right)+\Delta A\left(\eta_{t_{k}}, t_{k}\right) B\left(\eta_{t_{k}}\right)=B_{i} \quad A_{d}\left(\eta_{t_{k}}\right)=A_{d i} \\
\Delta A_{d}\left(\eta_{t_{k}}, t_{k}\right)=\Delta A_{d i}\left(t_{k}\right) \quad A_{d i}\left(t_{k}\right)=A_{d i}+\Delta A_{d i}\left(t_{k}\right)
\end{gathered}
$$

The norm-bounded parameter uncertainties $\Delta A_{i}\left(t_{k}\right)$ and $\Delta A_{d i}\left(t_{k}\right)$ are time-varying matrices with appropriate dimension, which are defined as follow: 


$$
\begin{aligned}
& {\left[\Delta A_{i}\left(t_{k}\right) \Delta A_{d i}\left(t_{k}\right)\right]=D_{i} F_{i}\left(t_{k}\right)\left[E_{1 i} E_{2 i}\right]} \\
& F_{i}^{T}\left(t_{k}\right) F_{i}\left(t_{k}\right) \leq I, \quad \forall t_{k} \geq 0
\end{aligned}
$$

Where $D_{i}, E_{1 i}$ and $E_{2 i}$ are known constant real matrices with appropriate dimension, and $F_{i}\left(t_{k}\right)$ is unknown time-varying matrix .

Definition 1 A jump delta operator system is stochastic asymptotically stable in delta domain, if the following conditions hold:

(i) $V\left(x\left(t_{k}\right)\right) \geq 0$,with equality if and only if $x\left(t_{k}\right)=0$;

(ii) $\sigma V\left(x\left(t_{k}\right)\right)=\frac{1}{T}\left[E\left[V\left(x\left(t_{k}+T\right)\right)\right]-V\left(x\left(t_{k}\right)\right)\right]$,

where $V\left(x\left(t_{k}\right)\right)$ is a Lyapunov function in delta domain.

Before ending this section, the following lemmas will be recalled to prove our main results in the next section.

Lemma 1 [9] For any constant positive semi-definite symmetric matrix $W$, two positive integers $r$ and $r_{0}$ satisfying $r \geq r_{0} \geq 1$, the following inequality holds

$$
\left(\sum_{i=r_{0}}^{r} x(i)\right)^{T} W\left(\sum_{i=r_{0}}^{r} x(i)\right) \leq\left(r-r_{0}+1\right) \sum_{i=r_{0}}^{r} x^{T}(i) W x(i)
$$

Lemma 2 [10] For some given matrices $\gamma, D$ and $E$ of appropriate dimension and with $\gamma$ symmetric, then

$$
\gamma+D F\left(t_{k}\right) E+E^{T} F^{T}\left(t_{k}\right) D^{T}<0
$$

where $F\left(t_{k}\right)$ is given as in (2), if and only if there exists a scalar $\varepsilon>0$ such that

$$
\gamma+\varepsilon D D^{T}+\varepsilon^{-1} E^{T} E<0
$$

\section{MAIN RESULTS}

The following control law is employed to deal with the problem of stabilization via state feedback:

$$
u\left(t_{k}\right)=K\left(t_{k}\right) x\left(t_{k}\right)
$$

We denote $K\left(t_{k}\right)$ by $K_{i}$, where $K\left(t_{k}\right)$ is the state feedback controller gain to be determined such that the closed-loop system is asymptotically stable for any $d_{m} \leq d(k) \leq d_{M}$. Consider system (1) associated with the control law (4), then the resulting closed-loop system can be expressed as follows:

$$
\begin{aligned}
\delta x\left(t_{k}\right)= & \left(A\left(\eta_{t_{k}}\right)+\Delta A\left(\eta_{t_{k}}, t_{k}\right)+B\left(\eta_{t_{k}}\right) K_{i}\right) x\left(t_{k}\right) \\
& +\left(A_{d}\left(\eta_{t_{k}}\right)+\Delta A_{d}\left(\eta_{t_{k}}, t_{k}\right)\right) x\left(t_{k}-d(k)\right)
\end{aligned}
$$

In this section, we let $A_{K i}\left(t_{k}\right)=A_{i}\left(t_{k}\right)+B_{i} K_{i}$. An LMI approach is developed for designing the desired state feedback controller. The following theorem presents a delay-ranged-dependent result given in terms of LMIs.

Theorem 1 Considering the uncertain closed-loop system (5), if there exist matrices $X_{i j}>0, X_{i}>0, Z_{i}>0, M_{i}>0$, $N_{i}>0$ and $Y_{i}$, as well as a positive scalar $\varepsilon_{i}$ such that the following LMI holds:

$$
\left[\begin{array}{ccccc}
\Pi(1,1) & \Pi(1,2) & A_{d i} X_{i} & 0 & 0 \\
* & \Pi(2,2) & A_{d i} X_{i} & \frac{1}{d_{M}} N_{i} & E_{1 i}^{T} \\
* & * & -M_{i} & 0 & E_{2 i}^{T} \\
* & * & * & -Z_{i}-\frac{1}{d_{M}} N_{i} & 0 \\
* & * & * & * & -\varepsilon_{i} I
\end{array}\right]<0
$$

with

$$
\begin{aligned}
\Pi(1,1) & =T^{2} \sum_{j=1}^{s} \lambda_{i j} X_{i j}+(T-2) X_{i}+d_{M} N_{i}+\varepsilon_{i} D_{i}^{T} D_{i} \\
\Pi(1,2) & =T \sum_{j=1}^{s} \lambda_{i j} X_{i j}+\left(A_{i} X_{i}+B_{i} Y_{i}\right)+\varepsilon_{i} D_{i}^{T} D_{i} \\
\Pi(2,2) & =\sum_{j=1}^{s} \lambda_{i j} X_{i j}+\left(A_{i} X_{i}+B_{i} Y_{i}\right)^{T}+\left(A_{i} X_{i}+B_{i} Y_{i}\right) \\
& +\left(d_{M}-d_{m}+T+1\right) M_{i}+Z_{i}-\frac{1}{d_{M}} N_{i}+\varepsilon_{i} D_{i}^{T} D_{i}
\end{aligned}
$$

Proof Construct the Lyapunov-Krasovskii functional in delta domain as follows:

$$
V\left(t_{k}\right)=V_{1}\left(t_{k}\right)+V_{2}\left(t_{k}\right)+V_{3}\left(t_{k}\right)+V_{4}\left(t_{k}\right)+V_{5}\left(t_{k}\right)
$$

with

$$
\begin{aligned}
& V_{1}\left(t_{k}\right)=x^{T}\left(t_{k}\right) P\left(\eta_{t_{k}}\right) x\left(t_{k}\right) \quad V_{2}\left(t_{k}\right)=T \sum_{i=1}^{\hat{n}} x^{T}\left(t_{k}-i T\right) Q x\left(t_{k}-i T\right) \\
& V_{3}\left(t_{k}\right)=T \sum_{i=1}^{n_{M}} x^{T}\left(t_{k}-i T\right) S x\left(t_{k}-i T\right) \\
& V_{4}\left(t_{k}\right)=T^{2} \sum_{i=n_{m}}^{n_{M}} \sum_{j=1}^{i} x^{T}\left(t_{k}-j T\right) Q x\left(t_{k}-j T\right) \\
& V_{5}\left(t_{k}\right)=\sum_{i=n_{m}}^{n_{M}} \sum_{j=1}^{i} e^{T}\left(t_{k}-j T\right) \operatorname{Re}\left(t_{k}-j T\right)
\end{aligned}
$$

where $e(j)=x(j)-x(j+T)$, so there exist $\delta x(j)=-e(j) / T$ and $e(t-i T)=x(t-i T)-x(t-(i-1) T)$. The delta operator of 
the stochastic process $\left\{x\left(t_{k}\right), \eta_{t_{k}}, t_{k} \geq 0\right\}$, acting on $V\left(x, t_{k}, \eta_{t_{k}}\right)$ at the point $\left\{x, t_{k}, \eta_{t_{k}}=i\right\}$ can be expressed as follows:

$$
\sigma\left(V\left(t_{k}\right)\right)=\frac{E\left[V\left(t_{k}+T\right)\right]-V\left(t_{k}\right)}{T}
$$

Let the mode at the time $t_{k}$ be $i$, that is $\eta_{t_{k}}=i$. Recall that at the time $t_{k}+T$, the system may jump to any mode $\eta_{t_{k}+T}=j$. By letting $E\left(P_{j}\right)=T \sum_{j=1}^{s} \lambda_{i j} P_{j}+P_{i}$ and taking the stochastic delta operator manipulation of along the trajectory of system (5) we can obtain:

$$
\begin{array}{r}
\sigma V_{1}\left(t_{k}\right)=\frac{1}{T}\left\{E\left[x^{T}\left(t_{k}+T\right) P_{i} x\left(t_{k}+T\right)\right]-x^{T}\left(t_{k}\right) P_{i} x\left(t_{k}\right)\right\} \\
=\sum_{j=1}^{s} \lambda_{i j} x^{T}\left(t_{k}+T\right) P_{j} x\left(t_{k}+T\right) \\
+\frac{1}{T}\left[x^{T}\left(t_{k}+T\right) P_{i} x\left(t_{k}+T\right)-x^{T}\left(t_{k}\right) P_{i} x\left(t_{k}\right)\right] \\
=T^{2} \sum_{j=1}^{s} \lambda_{i j} \delta^{T}\left(x\left(t_{k}\right)\right) P_{j} \delta\left(x\left(t_{k}\right)\right) \\
+T \sum_{j=1}^{s} \lambda_{i j} \delta^{T} x\left(t_{k}\right) P_{j} x\left(t_{k}\right)+T \sum_{j=1}^{s} \lambda_{i j} x^{T}\left(t_{k}\right) P_{j} \delta\left(x\left(t_{k}\right)\right) \\
+\sum_{j=1}^{s} \lambda_{i j} x^{T}\left(t_{k}\right) P_{j} x\left(t_{k}\right)+T \delta^{T}\left(x\left(t_{k}\right)\right) P_{i} \delta\left(x\left(t_{k}\right)\right) \\
+\delta^{T}\left(x\left(t_{k}\right)\right) P_{i} x\left(t_{k}\right)+x^{T}\left(t_{k}\right) P_{i} \delta\left(x\left(t_{k}\right)\right)
\end{array}
$$

Taking the stochastic delta operator manipulation of $V_{2}\left(t_{k}\right)$, $V_{3}\left(t_{k}\right)$ and $V_{4}\left(t_{k}\right)$, it can be obtained that

$$
\begin{aligned}
\sigma V_{2}\left(t_{k}\right)= & \frac{1}{T}\left[T \sum_{i=1}^{\hat{n}} x^{T}\left(t_{k}-(i-1) T\right) Q x\left(t_{k}-(i-1) T\right)\right. \\
& \left.-T \sum_{i=1}^{\hat{n}} x^{T}\left(t_{k}-i T\right) Q x\left(t_{k}-i T\right)\right] \\
\leq & x^{T}\left(t_{k}\right) Q x\left(t_{k}\right)-x^{T}\left(t_{k}-d(k)\right) Q x\left(t_{k}-d(k)\right) \\
& +T \sum_{i=n_{m}}^{n_{M}} x^{T}\left(t_{k}-i T\right) Q x\left(t_{k}-i T\right)
\end{aligned}
$$$$
\sigma V_{3}\left(t_{k}\right)=x^{T}\left(t_{k}\right) S x\left(t_{k}\right)-x^{T}\left(t_{k}-d_{M}\right) S x\left(t_{k}-d_{M}\right)
$$

$$
\begin{aligned}
\sigma V_{4}\left(t_{k}\right)= & T \sum_{i=n_{m}}^{n_{M}}\left[\sum_{j=1}^{i} x^{T}\left(t_{k}-(j-1) T\right) Q x\left(t_{k}-(j-1) T\right)\right. \\
& \left.-\sum_{j=1}^{i} x^{T}\left(t_{k}-j T\right) Q x\left(t_{k}-j T\right)\right] \\
= & T\left(n_{M}-n_{m}+1\right) x^{T}\left(t_{k}\right) Q x\left(t_{k}\right)-T \sum_{i=n_{m}}^{n_{M}} x^{T}\left(t_{k}-i T\right) Q x\left(t_{k}-i T\right) \\
= & \left(d_{M}-d_{m}+T\right) x^{T}\left(t_{k}\right) Q x\left(t_{k}\right)-T \sum_{i=n_{m}}^{n_{M}} x^{T}\left(t_{k}-i T\right) Q x\left(t_{k}-i T\right)
\end{aligned}
$$

Taking the delta operator manipulation of $V_{5}\left(t_{k}\right)$, and using Lemma 1 , result in

$$
\begin{aligned}
\sigma V_{5}\left(t_{k}\right)= & \frac{1}{T}\left[\sum_{i=1}^{n_{M}} \sum_{j=1}^{i} e\left(t_{k}-(j-1) T\right) \operatorname{Re}\left(t_{k}-(j-1) T\right)\right. \\
- & \left.\sum_{i=1}^{n_{M}} \sum_{j=1}^{i} e\left(t_{k}-j T\right) \operatorname{Re}\left(t_{k}-j T\right)\right] \\
\leq & \frac{n_{M}}{T} e^{T}\left(t_{k}\right) \operatorname{Re}\left(t_{k}\right)-\frac{1}{n_{M} T}\left[\sum_{i=1}^{n_{M}} e\left(t_{k}-i T\right)\right]^{T} R\left[\sum_{i=1}^{n_{M}} e\left(t_{k}-i T\right)\right] \\
= & -\frac{1}{d_{M}}\left[x\left(t_{k}-d_{M}\right)-x\left(t_{k}\right)\right]^{T} R\left[x\left(t_{k}-d_{M}\right)-x\left(t_{k}\right)\right] \\
& +d_{M} \delta^{T}\left(x\left(t_{k}\right)\right) R \delta\left(x\left(t_{k}\right)\right)
\end{aligned}
$$

For the positive definite real matrix $P_{i}$, one has that

$$
\begin{aligned}
0= & -2 \delta^{T}\left(x\left(t_{k}\right)\right) P_{i}\left[\delta\left(x\left(t_{k}\right)\right)-A_{K i} x\left(t_{k}\right)-A_{d i}\left(t_{k}\right) x\left(t_{k}-d(k)\right)\right] \\
= & -2 \delta^{T}\left(x\left(t_{k}\right)\right) P_{i} \delta\left(x\left(t_{k}\right)\right)+2 \delta^{T}\left(x\left(t_{k}\right)\right) P_{i} A_{K i} x\left(t_{k}\right) \\
& +2 \delta^{T}\left(x\left(t_{k}\right)\right) P_{i} A_{d i}\left(t_{k}\right) x\left(t_{k}-d(k)\right)
\end{aligned}
$$

Finally, it follows from (7)-(13) that

$$
\sigma V\left(t_{k}\right)<\xi^{T}\left(t_{k}\right) \Pi_{1} \zeta\left(t_{k}\right)<0
$$

where

$$
\begin{aligned}
\xi^{T}\left(t_{k}\right) & =\left[\begin{array}{cccc}
\delta^{T}\left(x\left(t_{k}\right)\right) & x^{T}\left(t_{k}\right) & x^{T}\left(t_{k}-d(k)\right) & x^{T}\left(t_{k}-d_{M}\right)
\end{array}\right] \\
\Pi_{1} & =\left[\begin{array}{cccc}
\Pi_{1}(1,1) & \Pi_{1}(1,2) & P_{i} A_{d i}\left(t_{k}\right) & 0 \\
* & \Pi_{1}(2,2) & P_{i} A_{d i}\left(t_{k}\right) & \frac{1}{d_{M}} R \\
* & * & -Q & 0 \\
* & * & * & -S-\frac{1}{d_{M}} R
\end{array}\right]
\end{aligned}
$$

where 


$$
\begin{array}{lr}
\Pi_{1}(1,1)=T^{2} \sum_{j=1}^{s} \lambda_{i j} P_{j}+(T-2) P_{i}+d_{M} R & \Pi_{2}(1,1)=T^{2} \sum_{j=1}^{s} \lambda_{i j} P_{j}+(T-2) P_{i}+d_{M} R+\varepsilon_{i} P_{i} D_{i} D_{i}^{T} P_{i} \\
\Pi_{1}(1,2)=T \sum_{j=1}^{s} \lambda_{i j} P_{j}+P_{i} A_{K i}\left(t_{k}\right) & \Pi_{2}(1,2)=T \sum_{j=1}^{s} \lambda_{i j} P_{j}+P_{i}\left(A_{i}+B_{i} K_{i}\right)+\varepsilon_{i} P_{i} D_{i} D_{i}^{T} P_{i} \\
\Pi_{1}(2,2)=\sum_{j=1}^{s} \lambda_{i j} P_{j}+A_{K i}^{T}\left(t_{k}\right) P_{i}+P_{i} A_{K i}\left(t_{k}\right)+\left(d_{M}-d_{m}+T+1\right) Q+S-\frac{1}{d_{M}} R \Pi_{2}(2,2)=\sum_{j=1}^{s} \lambda_{i j} P_{j}+\left(A_{i}+B_{i} K_{i}\right)^{T} P_{i}+P_{i}\left(A_{i}+B_{i} K_{i}\right)
\end{array}
$$

From (2) and (14), we get that

$$
\Pi_{1}=\gamma_{1}+\eta_{1 i} F\left(t_{k}\right) \varsigma_{1 i}+\varsigma_{1 i}{ }^{T} F^{T}\left(t_{k}\right) \eta_{1 i}{ }^{T}<0
$$

Where

$$
\begin{gathered}
\gamma_{1}=\left[\begin{array}{cccc}
\gamma_{1}(1,1) & \gamma_{1}(1,2) & P_{i} A_{d i} & 0 \\
* & \gamma_{1}(2,2) & P_{i} A_{d i} & \frac{1}{d_{M}} R \\
* & * & -Q & 0 \\
* & * & * & -S-\frac{1}{d_{M}} R
\end{array}\right] \\
\eta_{1 i}=\left[\begin{array}{llll}
D_{i}^{T} P_{i} & D_{i}^{T} P & 0 & 0
\end{array}\right]_{. .}^{T} S_{1 i}=\left[\begin{array}{llll}
0 & E_{1 i} & E_{2 i} & 0
\end{array}\right]
\end{gathered}
$$

with

$$
\begin{aligned}
\gamma_{1}(1,1)= & T^{2} \sum_{j=1}^{s} \lambda_{i j} P_{j}+(T-2) P_{i}+d_{M} R \\
\gamma_{1}(1,2)= & T \sum_{j=1}^{s} \lambda_{i j} P_{j}+P_{i}\left(A_{i}+B_{i} K_{i}\right) \\
\gamma_{1}(2,2)= & \sum_{j=1}^{s} \lambda_{i j} P_{j}+\left(A_{i}+B_{i} K_{i}\right)^{T} P_{i}+P_{i}\left(A_{i}+B_{i} K_{i}\right) \\
& +\left(d_{M}-d_{m}+T+1\right) Q+S-\frac{1}{d_{M}} R
\end{aligned}
$$

By Lemma 2, there exists a scalar $\varepsilon_{i}>0$ such that the (15) equal to the following inequality

$$
\Pi_{1}=\gamma_{1}+\varepsilon_{i} \eta_{1 i} \eta_{1 i}^{T}+\varepsilon_{i}^{-1} \varsigma_{1 i}^{T} \varsigma_{1 i}<0
$$

Using Schur's complement, $\Pi_{1}<0$ can be changed to $\Pi_{2}<0$, where

$$
\Pi_{2}=\left[\begin{array}{ccccc}
\Pi_{2}(1,1) & \Pi_{2}(1,2) & P_{i} A_{d i} & 0 & 0 \\
* & \Pi_{2}(2,2) & P_{i} A_{d i} & \frac{1}{d_{M}} R & E_{1 i}{ }^{\mathrm{T}} \\
* & * & -Q & 0 & E_{2 i}{ }^{\mathrm{T}} \\
* & * & * & -S-\frac{1}{d_{M}} R & 0 \\
* & * & * & * & -\varepsilon_{i} I
\end{array}\right]
$$

with

$$
+\left(d_{M}-d_{m}+T+1\right) Q+S-\frac{1}{d_{M}} R+\varepsilon_{i} P_{i} D_{i} D_{i}^{T} P_{i}
$$

Then pre- and post-multiplying two sides of $\Pi_{2}<0$ by $\operatorname{diag}\left\{\begin{array}{lllll}P_{i}^{-1} & P_{i}^{-1} & P_{i}^{-1} & P_{i}^{-1} & I\end{array}\right\}$ respectively, and letting

$$
X_{i j}=P_{i}^{-1} P_{j} P_{i}^{-1}, X_{i}=P_{i}^{-1}, Y_{i}=K_{i} X_{i}=K P_{i}^{-1}, Z_{i}=P_{i}^{-1} S P_{i}^{-1} \text {, the }
$$
inequality $\Pi_{2}<0$, is inequality to $\Pi<0$.

From Definition 1, it can easily concluded that the delta operator system (1) with $u\left(t_{k}\right)=K_{i} x\left(t_{k}\right)$ is stochastic asymptotically stable if (6) holds.

\section{IV. NuMERAL EXAMPLE}

The time-varying delayed delta operator system (1) with $i=1,2$ is considered. The parameters are described by

$$
\begin{aligned}
& A_{1}=\left[\begin{array}{cc}
-2 & 4 \\
1 & -5
\end{array}\right], A_{d 1}=\left[\begin{array}{cc}
0.2 & 0.1 \\
0.2 & -0.3
\end{array}\right] D_{1}=\left[\begin{array}{l}
0.1 \\
0.1
\end{array}\right], B_{1}=\left[\begin{array}{l}
0.2 \\
0.1
\end{array}\right] \\
& E_{11}=\left[\begin{array}{ll}
0.04 & 0.02
\end{array}\right], E_{21}=\left[\begin{array}{ll}
0.03 & 0.02
\end{array}\right] \\
& A_{2}=\left[\begin{array}{cc}
-3 & 2 \\
5 & -4
\end{array}\right], A_{d 2}=\left[\begin{array}{cc}
-0.1 & 0.3 \\
0.5 & -0.4
\end{array}\right] D_{2}=\left[\begin{array}{l}
0.3 \\
0.2
\end{array}\right], B_{2}=\left[\begin{array}{l}
0.3 \\
0.4
\end{array}\right] \\
& E_{12}=\left[\begin{array}{ll}
0.02 & 0.01
\end{array}\right], E_{22}=\left[\begin{array}{ll}
0.03 & 0.01
\end{array}\right], \Lambda=\left[\begin{array}{ll}
0.2 & 0.8 \\
0.4 & 0.6
\end{array}\right]
\end{aligned}
$$

In order to design a state feedback controller for delta operator system (4), we first choose $d_{m}=0.1$, and $d_{M}=0.2$, $T=0.1$.Then, using Matlab LMI Control Toolbox to solve the LMIs in (6), the solution is obtained as follows:

$$
\begin{aligned}
& X_{11}=\left[\begin{array}{cc}
112.3335 & 9.6267 \\
9.6267 & 137.3710
\end{array}\right], X_{12}=\left[\begin{array}{ll}
91.8778 & -4.6316 \\
-4.6316 & 80.5278
\end{array}\right], \\
& X_{1}=\left[\begin{array}{ll}
106.3393 & 24.9094 \\
24.9094 & 11.5336
\end{array}\right], \quad Y_{1}=\left[\begin{array}{ll}
235.4631 & 782.8828
\end{array}\right], \\
& Z_{1}=\left[\begin{array}{ll}
79.8792 & 11.4970 \\
11.4970 & 61.6986
\end{array}\right], \\
& N_{1}=\left[\begin{array}{cc}
14.8401 & 1.7083 \\
1.7083 & 11.0335
\end{array}\right], \quad M_{1}=\left[\begin{array}{ll}
96.2829 & 15.7631 \\
15.7631 & 67.6810
\end{array}\right], \\
& K_{1}=\left[\begin{array}{ll}
-27.6987 & 127.6996
\end{array}\right], \quad X_{21}=\left[\begin{array}{ll}
1.1682 & 0.0806 \\
0.0806 & 1.2283
\end{array}\right], \\
& X_{22}=\left[\begin{array}{cc}
3.5684 & -0.6033 \\
-0.6033 & 3.1234
\end{array}\right], X_{2}=\left[\begin{array}{ll}
0.3146 & 0.2474 \\
0.2474 & 0.4303
\end{array}\right], \\
& M_{2}=\left[\begin{array}{ll}
0.7630 & 0.6862 \\
0.6862 & 1.6950
\end{array}\right],
\end{aligned}
$$




$$
\begin{aligned}
Y_{2} & =\left[\begin{array}{ll}
-0.1525 & 1.5228
\end{array}\right], & Z_{2} & =\left[\begin{array}{ll}
0.7271 & 0.5026 \\
0.5026 & 1.0724
\end{array}\right], \\
\varepsilon_{2} & =0.1181, & K_{2} & =\left[\begin{array}{ll}
-5.9623 & 6.9669
\end{array}\right]
\end{aligned}
$$

\section{CONCLUSION}

In this paper, a solution to the robust stabilization controllers design problem for a class of Markovian jump delta operator systems is revealed. A numerical example shows the availability of the design method.

\section{ACKNOWLEDGMENT}

This work is supported by Natural Science Foundation of Fujian Province under Grant 2014J01222 and Project of Creative Team of Fujian Normal University: Nonlinear analysis and its applications (IRTL1206).

\section{REFERENCES}

[1] G.C. Goodwin, R. Lozano Leal, D. Q. Mayne, R. H. Middleton, "Rapprochement between continuous and discrete model reference adaptive control". Automatica, 22(2): 199-207, 1986.

[2] S. Chen, R. H. Istepanian, J. Wu, J. Chu, "Comparative study on optimizing closed-loop stability bounds of finite-precision controller structures with shift and delta operators". Systems and Control Letter, 40(3): 153-163, 2000

[3] D. Li, S. Fei, "Robust stabilization for delta operator based systems". Fuzzy Systems and Mathematics, 19(2): 140- 145, 2005.

[4] Z. Xiang, Q. Chen, R. Zhang, "Robust stability analysis and control for fuzzy systems with uncertainties using the delta operator". Control and Decision, 18(6): 720- 723, 2003.

[5] W. Ebert, "Optimal filtered predictive control a delta operator approach". Systems and Control Letters, 42(1): 69- 80, 2001.

[6] M. Basin, J. Rodriguez-Gonzalez, "A closed form optimal control for linear systems with equal state and input delays". Automatica, 41(5): 915920, 2005.

[7] M. Basin, J. Rodriguez-Conzalez, L. Fridman, "Optimal and Robust control for linear state-delay systems". Journal of the Franklin Institute, 344(6): 830- 845, 2007.

[8] L. Xie, L. Wang, de Souza, "Robust control of a class of uncertain nonlinear system". Systems and Control Letters, 19: 139-149, 1992.

[9] H. Yang, Y. Xia, P. Shi, B. Liu, "Guaranteed cost control of networked control systems based on delta operator Kalman filter, Int.J. Adapt". Control Signal Process 27(8): 701-701, 2013.

[10] H. Yang, Y. Xia , P. Shi, M. Fu, "A novel delta operator Kalman filterdesign and convergence analysis", IEEE Trans. Circuits Syst. I: Regular Papers 58 (10): 2458-2468, 2011.

[11] L. Hu, P. Shi, Y. Cao, "Delay-dependent filtering design for time-delay system with Markovian jumping parameters".Int. J. Adapt. Contr.Sig.Proc.,21(5):434-448,2007. 